\title{
CIBERGEOGRAFÍA O EL SALTO DE LO TERRENAL A LO DIGITAL: LAS POSIBILIDADES Y RIESGOS DE UN MUNDO EN RED $\infty$
}

\section{CRISTIAN BARRÍA HUIDOBRO*}

"Dime por dónde navegas y te diré quién te..."

\begin{abstract}
RESUMEN
Internet representa los cimientos de la sociedad interconectada de hoy, en donde además de remodelar la forma en que nos relacionamos con el mundo, también plantea desafíos a nuestra representación mental y gráfica de los espacios físicos. La integración de nociones virtuales en casi la totalidad de las interacciones en la sociedad moderna, ha evidenciado la necesidad de replantear algunas visiones tradicionales en diversos campos del saber. En este estudio abordamos el caso de la cibergeografía, disciplina emergente que si bien puede trazar sus raíces a los trabajos cartográficos del siglo XIX, no ha sido sino hasta las últimas décadas que ha visto un sustantivo incremento en la complejidad de las representaciones lógicas que requieren ser plasmadas en mapas, los cuales exigen una armonía con los componentes físicos que interactúan de alguna u otra forma, con el mundo virtual. A pesar de que, como casi todo el fenómeno "ciber", la cibergeografía no cuenta con acuerdos $100 \%$ transversales a nivel de definiciones conceptuales, sí se han hecho avances epistemológicos importantes, llevando a la propuesta de ciertos criterios a la hora de plasmar cartográficamente los distintos niveles del ciberespacio, sin perder su relación con los elementos geográficamente tradicionales que componen el mundo físico.
\end{abstract}

Palabras clave: Cibergeografía; ciberespacio; cibercrimen; ciberseguridad.

\section{CYBERGEOGRAPHY. A LEAP FROM THE PHYSICAL TO A DIGITAL WORLD: THE POSSIBILITIES AND RISKS OF A NETWORKED WORLD}

\footnotetext{
ABSTRACT

The Internet represents the foundations of today's interconnected society, a place where we have changed the way in which we interact

× Fecha de recepción: 210120 - Fecha de aceptación: 130820.

- Postdoctorado (e) en Alta Investigación en Educación Intercultural. Doctor en Ingeniería Informática, Magíster en Ciencias de la Ingeniería Informática, y Magíster en Planificación y Gestión Educacional, Licenciado en Informática y Licenciado en Ciencias de la Ingeniería, cuenta con la Ingeniería en Informática, e Ingeniería en Administración, Director del Centro de Investigación en Ciberseguridad de la Universidad Mayor. cristian.barria@mayor.cl ORCID: https:/Lorcid.orgL0000-0002-5840-7407
} 
with one another, it also poses challenges to our mental and graphic representation of physical spaces. The integration of virtual notions in almost all interactions in modern society have become evident the need to reconsider some traditional visions in various fields of knowledge. This study revises cybergeography, an emerging discipline can be traced since the cartographic works of the 19th century. Only recently a substantial increase in the complexity of the logical representations which should be captured on maps, and they also demand a harmony with the physical components and the virtual world. Although, like most of the "cyber" phenomena, cybergeography does not have $100 \%$ transversal agreements at the level of conceptual definitions, important epistemological advances have been made, leading to the proposal of certain criteria when it comes to mapping cartographically the different levels of cyberspace, without losing its relationship with the geographically traditional elements that make up the physical world.

Key words: Cybergeography; cyberspace; cybercrime; cybersecurity.

\section{CIBERGEOGRAFIA OU O SALTO DA TERRA PARA O DIGITAL: AS POSSIBILIDADES E OS RISCOS DE UM MUNDO EM REDE}

\section{RESUMO}

A Internet representa os alicerces da sociedade interconectada de hoje, onde além de remodelar a forma como nos relacionamos com o mundo, também coloca desafios à nossa representação mental e gráfica dos espaços físicos. A integração de noções virtuais em quase todas as interações na sociedade moderna tem mostrado a necessidade de se repensar algumas visões tradicionais em vários campos do conhecimento. Neste estudo abordamos o caso da cibergeografia, uma disciplina emergente que, embora possa remontar às obras cartográficas do século XIX, não foi senão nas últimas décadas que assistiu a um aumento substancial da complexidade das representações lógicas que o requerem. ser capturados em mapas, que requerem harmonia com os componentes físicos que interagem de uma forma ou de outra com o mundo virtual. Apesar de, como quase todo o fenômeno "cibernético", a cibergeografia não ter acordos 100\% transversais ao nível das definições conceituais, se houve avanços epistemológicos importantes que levaram à proposição de determinados critérios no mapeamento cartográfico. os diferentes níveis do ciberespaço, sem perder sua relação com os elementos geograficamente tradicionais que compõem o mundo físico.

Palavras-chave: Cibergeografia; Ciberespaço; cibercrime; cíber segurança. 


\section{UN NUEVO CONTEXTO}

Según Luis Feliú Ortega ${ }^{1}$, hasta hace algunos años no existía un problema respecto de las definiciones "porque se seguía el método cartesiano y -los sistemas francés y alemán- cuyos idiomas son muy precisos. Sin embargo, en la actualidad, la influencia de las doctrinas anglosajonas, muy poco proclives a preocuparse por la precisión en el lenguaje y-con una- tendencia a utilizar la ambigüedad y los eufemismos en la utilización de determinados conceptos, han hecho que exista bastante confusión".

Es así como observamos que en el transcurso del tiempo suelen aparecer nuevos términos o bien uno antiguo adquiere un significado nuevo y espontáneamente se habla de él en todas partes. Ello ha sido sumamente visible en los últimos años, donde han surgido palabras de antiguo cuño a las que se ha añadido el sintagma "ciber" como un prefijo, transformándolas por este expediente en algo nuevo, aunque como veremos no tanto. Lo importante es que cualquier definición, como la de espacio, por ejemplo, no cambia por el hecho de que se le añada "ciber" al comienzo. Es decir, sigue siendo un espacio, solo que una región particular de él.

A pesar de lo anterior, es necesario hacer algunas precisiones en torno al tema que nos ocupa (que es la Cibergeografía), como es el caso de algunas definiciones que es importante tomar en cuenta. Comencemos por "Ciber". Para Stuart Umpledy y Eric Dent, el término aparece por primera vez en 1948, como título del libro "Cybernetics," de Norbert Wiener, profesor de Matemáticas del Instituto de Tecnología de Massachusetts, con un subtítulo que busca aclarar el alcance del ensayo: "Control y Comunicaciones en los Animales y las Máquinas" ${ }^{2}$. Es decir, esta nueva expresión se encuentra asociado a relaciones, a lo que hay entre, o lo que media la comunicación entre personas, o entre personas y máquinas.

La expresión "cibernética", por su parte, guarda relación con la electrónica. Ya durante la guerra de Vietnam la investigación en los campus universitarios, apoyados por el Departamento de Defensa, comenzó a volverse controversial (esto es, ¿̇se realizaba para prolongar la guerra o para terminarla?, etc.). Un resultado de dicha discusión fue la denominada enmienda Mansfield, según la cual los investigadores financiados por dicho organismo tenían que explicar la relevancia de sus investigaciones con respecto a la misión de las Fuerzas Armadas. Es así como los investigadores en inteligencia artificial, como una forma de justificar la financiación, crearon la idea de que en un futuro las batallas se librarían utilizando robots o sensores electrónicos. De ahí la razón por la que, en aquella época de mucha beligerancia, la cibernética contribuyó a cimentar la idea de un "campo de batalla electrónico"3.

Desde un punto de vista militar, para Van Creveld, "la cibernética y los computadoras

1 FELIÚ Ortega, Luis, 2012, La confusa terminología de la seguridad y la defensa. IEEE, Documento de opinión. 2012. p. 6.

2 UMPLEBY, Stuart A. y DENT, Eric B., 1999. The Origins and Purposes of Several Traditions in Systems Theory and Cybernetics. Cybernetics and Systems. 1999. Vol. 30, no. 2, pp. 79-103. DOI 10.1080/019697299125299. Informa UK Limited.

3 Ibíd. 
trajeron algo más que cambios en la administración, la logística, las comunicaciones, la inteligencia y las operaciones, [por cuanto ella] también ayudó a que un nuevo conjunto de personas, personas que pensaban la guerra -y que, por lo tanto, la planeaban, preparaban, libraban y evaluaban- pudieran hacerse cargo de ello con la ayuda de nuevos criterios y desde un punto de vista totalmente novedoso" ${ }^{4}$.

Pese a lo anterior, para autores como Brett Williams el término "ciber" "no debe ser usada como verbo ni debe emplearse como un sustantivo que puede valerse por sí

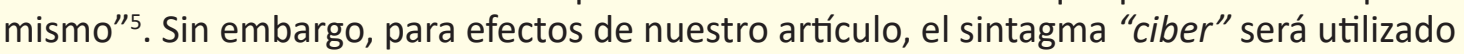
para todo aquello que guarde relación o interactúe en este curioso campo del ciberespacio, ya sea dentro de o en relación con él.

Por lo tanto, es en este marco conceptual donde viene a inscribirse nuestra mirada sobre la geografía, o más derechamente, sobre la Cibergeografía.

Esta disciplina es una especialidad muy reciente, que es más bien hija del siglo XXI, y cuyo nacimiento se produjo fundamentalmente entre el Reino Unido y los Estados Unidos. Se trata de un cambio de nivel para la ciencia geográfica. En este caso, su estudio comprende -además del territorio y sus múltiples ramas o acepciones- la naturaleza espacial de las redes de comunicaciones informáticas, incluyendo Internet y todo "lugar electrónico" que pueda existir entre los monitores computacionales ${ }^{678}$. En otras palabras, al ciberespacio.

Sin lugar a dudas, y sobre todo en la actualidad, resulta sorprende experimentar la instantaneidad con que día a día abordamos (y somos abordados por) las comunicaciones. Desde una perspectiva cibergeográfica, entonces, el asunto no deja de cobrar interés, por cuanto el ciberespacio, sin poseer una cartografía a la usanza geográfica, sí posee una propia en el contexto de las actuales tecnologías digitales. Para Carl Sagan, en el libro "La Conexión Cósmica" ${ }^{9}$, desde tiempos inmemoriales los seres humanos han reflexionado acerca del lugar que les toca ocupar en el universo y, por lo tanto, preguntarse qué lugar nos toca ocupar ahora en el ciberespacio no es una pregunta con sentido original. Lo que sí resulta original es el hecho de que este nuevo universo es, efectivamente, una creación humana.

Por lo mismo, la cibergeografía considera, entre otras, las relaciones socioespaciales

4 VAN CREVELD, Martin, 2010. Technology and war: From 2000 BC to the present. Simon and Schuster.

5 WILLIAMS, Brett, 2019. Cyberspace: What is it, where is it and who cares? Armed Forces Journal [online]. 2019. [Accessed 14 noviembre 2019]. Available from: http://armedforcesjournal.com/cyberspace-whatis-it-where-is-it-and-who-cares/

6 BUZAl, Gustavo, 2001. Cibergeografía y la destrucción del "mito" de la red mundial sin centro. Estudios socioterritoriales. 2001. Vol. 2, no. 2, pp. 9-24.

7 KITCHIN, Robert M., 1998. Towards geographies of cyberspace. Progress in Human Geography. 1998. Vol. 22, no. 3, pp. 385-406. DOI 10.1191/030913298668331585. SAGE Publications

8 RAMÍREZ Velázquez, Blanca Rebeca and LÓPEZ Levi, Liliana, 2015. Espacio, paisaje, región, territorio y lugar. 1. Geografía para el siglo XXI: Serie Textos universitarios.

9 SAGAN, Carl, 2000. Carl Sagan's cosmic connection. Cambridge: Cambridge University Press. 
que abarca claras líneas de estudio: la distribución espacial del equipamiento físico de las tecnologías de la información y las comunicaciones (TIC), los aspectos sociodemográficos de las nuevas comunidades virtuales, la visualización perceptiva de los nuevos espacios electrónicos, el estudio geográfico de los flujos comunicacionales, y las Tecnologías de la Información Geográfica (TIG) ${ }^{10}$. "Es en este último aspecto -el de las relaciones socioespaciales- donde el presente trabajo se focaliza, es decir, en la aparición de este nuevo tipo de geografía, de tal modo de conocer sus características, sus rasgos principales, hacia dónde debiera crecer o desarrollarse, y qué va a significar esto para todos nosotros".

Para introducirnos en esta temática observaremos que surgen componentes geográficos, los que relacionamos con la ubicación, ya sea en la tierra, el aire, el mar o el espacio. Luego, esta cibergeografía es el "lugar" donde se encuentran los elementos de las redes, cuyos componentes físicos comprenden el hardware, el software y la infraestructura (los cables, los sistemas Wireless, los enlaces electromagnéticos, los satelitales y los ópticos), que apoyan a las redes y a los conectores físicos (cables, radio frecuencia, routers, switches, servers y computadoras).

Por lo mismo, el ciberespacio es el campo donde traza y trazará sus "mapas" la cibergeografía. El ciberespacio, decía Ted Stevens, último senador de Alaska, "No es un camión. Es una serie de tubos". Una serie de tubos como aquellos por los que se enviaban mensajes de un lugar a otro en entidades cerradas como bancos o supermercados. Estos "tubos" de Stevens, pese a no representar exactamente lo que es el ciberespacio, ayudan a comprenderlo como un entramado, un tejido; una analogía, en buenas cuentas, que utilizan los expertos (no solo el antiguo senador) para describir las conexiones de datos ${ }^{11}$.

Para la geografía, por tanto, el ciberespacio viene a ser un "conjunto de medios y procedimientos basados en las Tecnologías de la Información y las Comunicaciones (TIC) configurados para la prestación de servicios. Está constituido por hardware, software, Internet, servicios de información y sistemas de control que garantizan la provisión de aquellos servicios esenciales para la actividad socioeconómica de cualquier estado, en especial aquellos ligados a sus infraestructuras críticas"12.

En consecuencia, es posible decir que la cibergeografía se refiere al estudio que comprenden las amplias relaciones entre lo real (espacio geográfico) y lo virtual (representación digital) ${ }^{13}$, que van a revolucionar (y ya lo están haciendo) el análisis geográfico del siglo XXI.

10 BUZAI, Gustavo D., 2001. Paradigma Geotecnológico, Geografía Global y CiberGeografía, la gran explosión de un universo digital en expansión. GeoFocus. Revista Internacional de Ciencia y Tecnología de la Información Geográfica. 2001. Vol. 1, pp. 24-48.

11 SINGER, P. W. and FRIEDMAN, Allan, 2014. Cybersecurity and cyberwar. New York: Oxford University Press.

12 SIERRA, Daniel, 2015. Las dos caras de la tecnología. CIBER Elcano [online]. $2015 . \quad$ No. 2 2. p. 15. [Accessed 14 november 2019]. Available from: http://www.realinstitutoelcano. org/wps/wcm/connect/6df2110047ec9efab8bbbe8dbc02bac7/Ciber_Elcano_Num2. pdf?MOD=AJPERES\&CACHEID $=1428393273980$

13 BUZAI, Gustavo, 2001. Loc. Cit. 


\section{REPRESENTACIONES GRÁFICAS DE LA CIBERGEOGRAFÍA}

Si bien la cibergeografía se alza como una disciplina relativamente nueva, el interés por la representación cartográfica de redes e interacciones puede trazarse muy atrás en el tiempo, hasta el desarrollo tecnológico de las telecomunicaciones, partiendo por el telégrafo y el teléfono durante el siglo XIX. En el año 1853, el cartógrafo Charles B. Barr produjo un detallado mapa (ver figura 1) que ilustra la red de telégrafos existente en esa época en los Estados Unidos ${ }^{14}$.

Figura 1

Telegraph stations in the United States, the Canadas \& Nova Scotia

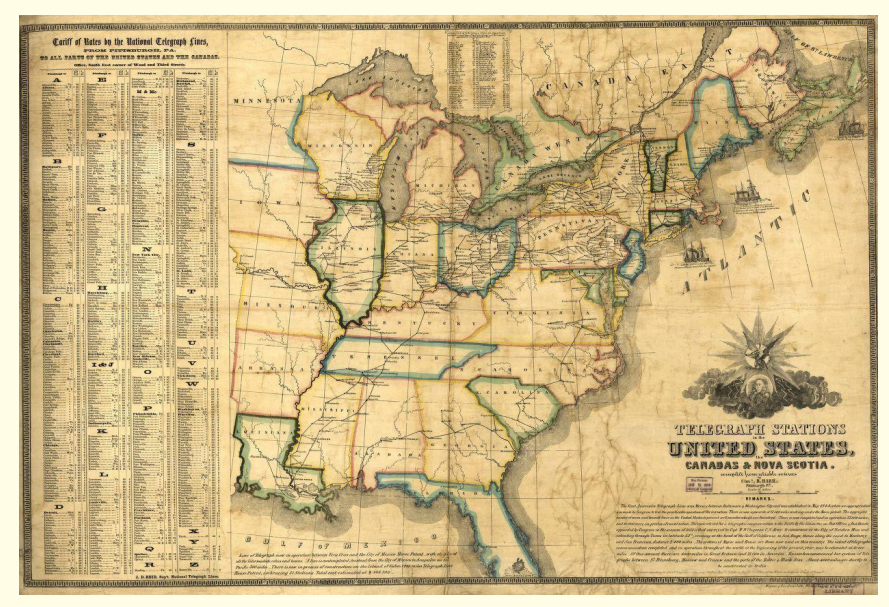

Fuente: Barr, 1853.

Su trabajo nos permite apreciar la lógica empleada en términos de simbología: las estaciones de telégrafos eran marcadas con un punto negro, mientras que las líneas que las interconectaban, con líneas negras. La red se superponía a un mapa tradicional que ilustraba los límites entre Estados, la presencia de ríos, entre otros aspectos, los cuales facilitan al lector la interpretación de la información, dando un sentido espacial a la ubicación de las estaciones. Esta simple, pero eficiente lógica, puede emplearse, por ejemplo, para producir un mapa de los Datacenters públicos y privados que prestan servicios a nuestras instituciones.

Por supuesto, en el caso de redes más complejas se debe recurrir a otras representaciones que permitan complementar la información provista en el mapa. Un ejemplo de esto es el mapa geográfico de ARPANET, la red computacional que sentó los cimientos de Internet: ella comenzó como un bosquejo a mano alzada, que ilustraba una simple red compuesta por un sistema computacional de tiempo compartido, y una interfaz de procesamiento de mensajes (ver figura 2) que fue evolucionando hasta una amplia representación de los distintos nodos computacionales distribuidos en el territorio

14 BARR, Chas. B., 1853. Telegraph stations in the United States, the Canadas \& Nova Scotia. [S.I] [Map] [online]. [image]. 1853. [Accessed 14 november 2019]. Available from: https://www.loc.gov/item/97683602/ 
estadounidense (ver figura 3$)^{15}{ }^{16}$. Cabe hacer notar que la alta concentración de nodos en ciertas zonas del territorio, delatan la densidad de establecimientos financiados por el gobierno, como también de instalaciones militares.

Figura 2

ARPANET first node sketch

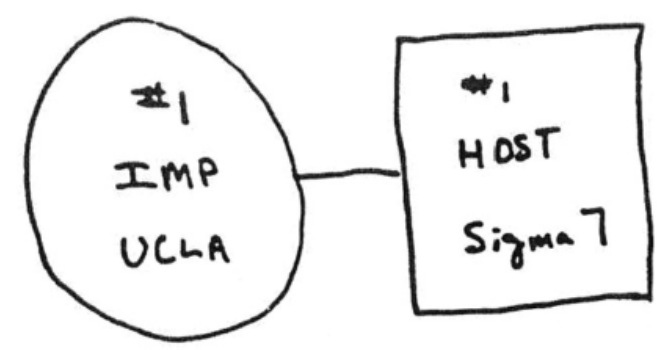

\section{THE ARPA NETWORK}

\section{SEPT 1969}

\section{NODE}

Fuente: Cerf and Kahn, 1990.

15 FIDLER, Bradley and CURRIE, Morgan, 2015. The Production and Interpretation of ARPANET Maps. IEEE Annals of the History of Computing. 2015. Vol. 37, no. 1, pp. 44-55. DOI 10.1109/mahc.2015.16. Institute of Electrical and Electronics Engineers (IEEE)

16 CERF, V. and KAHN, B., 1990. Selected ARPANET maps. Computer Communications Review (CCR). 1990. No. 20, pp. 81-110. 
Figura 3

ARPANET geographical map, 1977

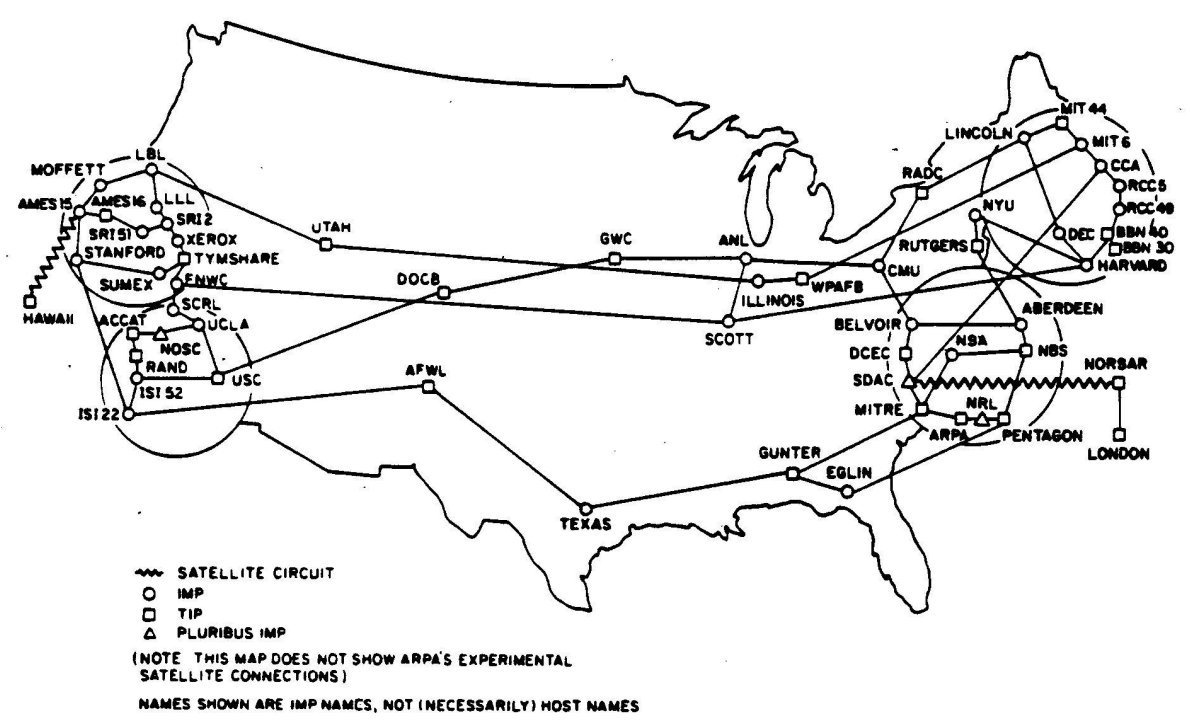

Fuente: Cerf and Kahn, 1990.

Hasta aquí, sin embargo, la cibergeografía no pasa de ser una representación de ubicaciones y conexiones superpuestas a un mapa tradicional. Por lo pronto, digamos que los aspectos a considerar son muchos más. Dodge y Kitchin realizaron uno de los trabajos más exhaustivos en la materia, a través de su Atlas del Ciberespacio, en el que se plantean distintas formas de representar los mapas cibergeográficos ${ }^{17}$.

Los autores proponen tres enfoques para estas representaciones, a saber: "el mapeo de infraestructura y tráfico, el mapeo de la red, y el mapeo de las conversaciones y la comunidad". Así como en la geografía tradicional un espacio específico puede utilizarse para una diversidad de mapas, en la cibergeografía la representación gráfica de un ciberespacio puntual puede abordarse de diferente manera. El enfoque hacia la infraestructura y tráfico apunta a proveer representaciones exactas de la ubicación física de una infraestructura de red. Este tipo de mapas resulta sumamente práctico, por la sencilla razón de que gran parte de la arquitectura de red no es habitualmente visible. Un Access Point cuya antena provee de Internet inalámbrico a una oficina, por ejemplo, está físicamente conectada a la infraestructura de red de dicha oficina, la cual a su vez está físicamente conectada a la infraestructura de red del proveedor de servicios de Internet (ISP) contratado. Esta conexión física se traduce en cables y dispositivos que habitualmente pasan bajo pisos flotantes, entretechos, tuberías, o cubresuelos. Lo que vemos, entonces, normalmente es el punto final de toda la infraestructura física, no su alcance real.

17 DODGE, Martin and KITCHIN, Rob, 2001. Atlas of cyberspace. Harlow: Pearson Education. 
En la figura 4 se ilustra un ejemplo de este tipo de mapeo, pero a escala global: se trata de un mapa de los cables submarinos de fibra óptica distribuidos por el mundo ${ }^{18}$.

Figura 4: Submarine Cable Map.

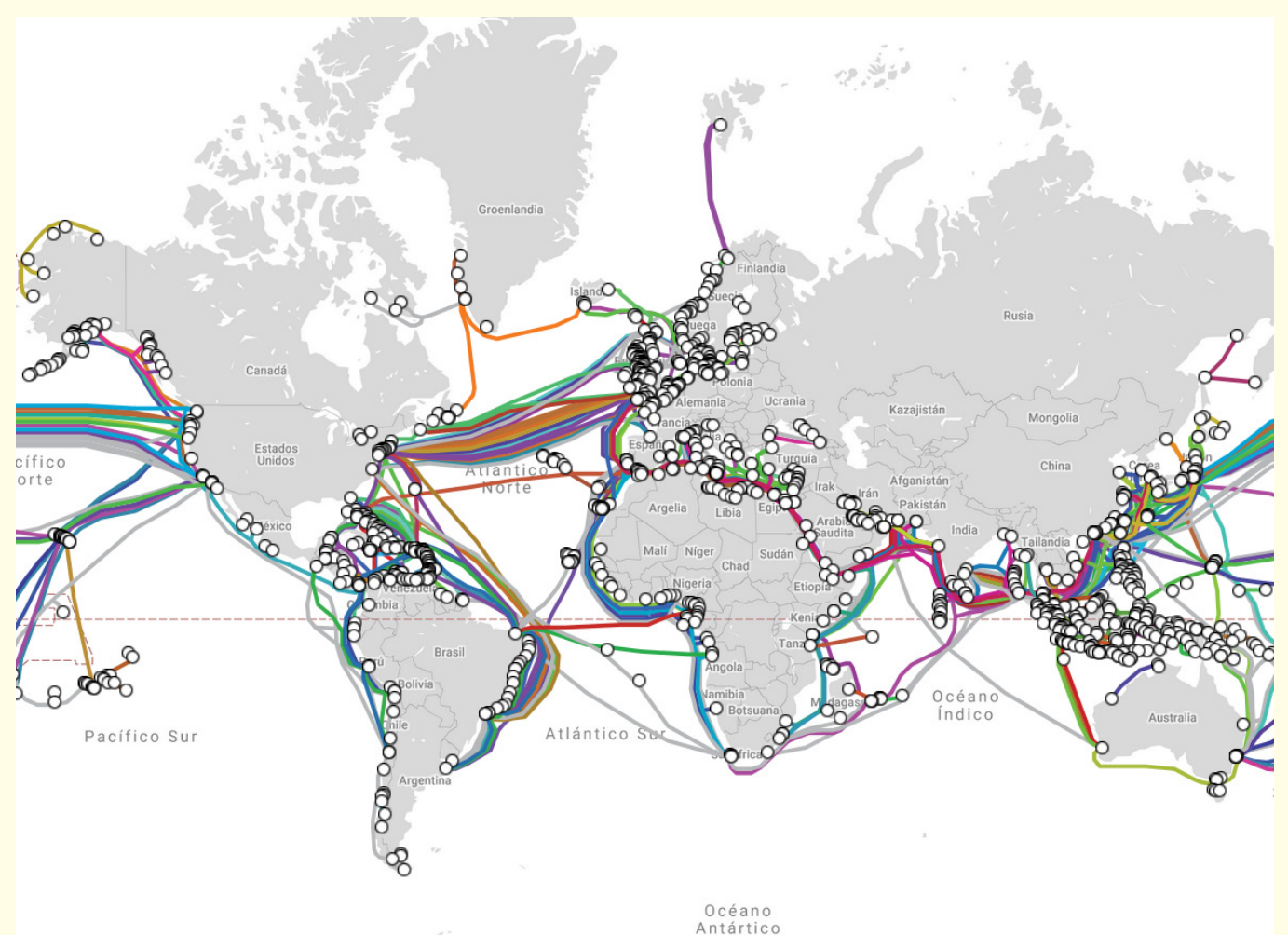

Fuente: https://www.submarinecablemap.com/

En el caso del Internet satelital, la cartografía no es estática, es dinámica, por lo mismo se debe considerar que está siempre en movimiento. Luego, un mapa estático solo permitirá obtener información de un instante específico en el tiempo.

En este sentido, una ilustración animada que vaya ajustando la información gráfica en tiempo real, resulta una opción mucho más apropiada, especialmente cuando se requiere contar con información actualizada para una rápida toma de decisiones. En la figura 5 se muestra una vista parcial de esta interfaz ${ }^{19}$, tal como la visualizaría un usuario que trabaje con actualizaciones de momento a momento.

18 Submarine Cable Map, [s. f.]. https://www.submarinecablemap.com/ [online], [Accessed 14 november 2019].

19 FORD, Dominic, 2019. Live World Map of Satellite Positions - In-The-Sky.org. In-the-sky.org [online]. 2019. [Accessed 14 noviembre 2019]. Available from: https://in-the-sky.org/satmap_worldmap.php 
Figura 5

Live Map of Satellite Positions

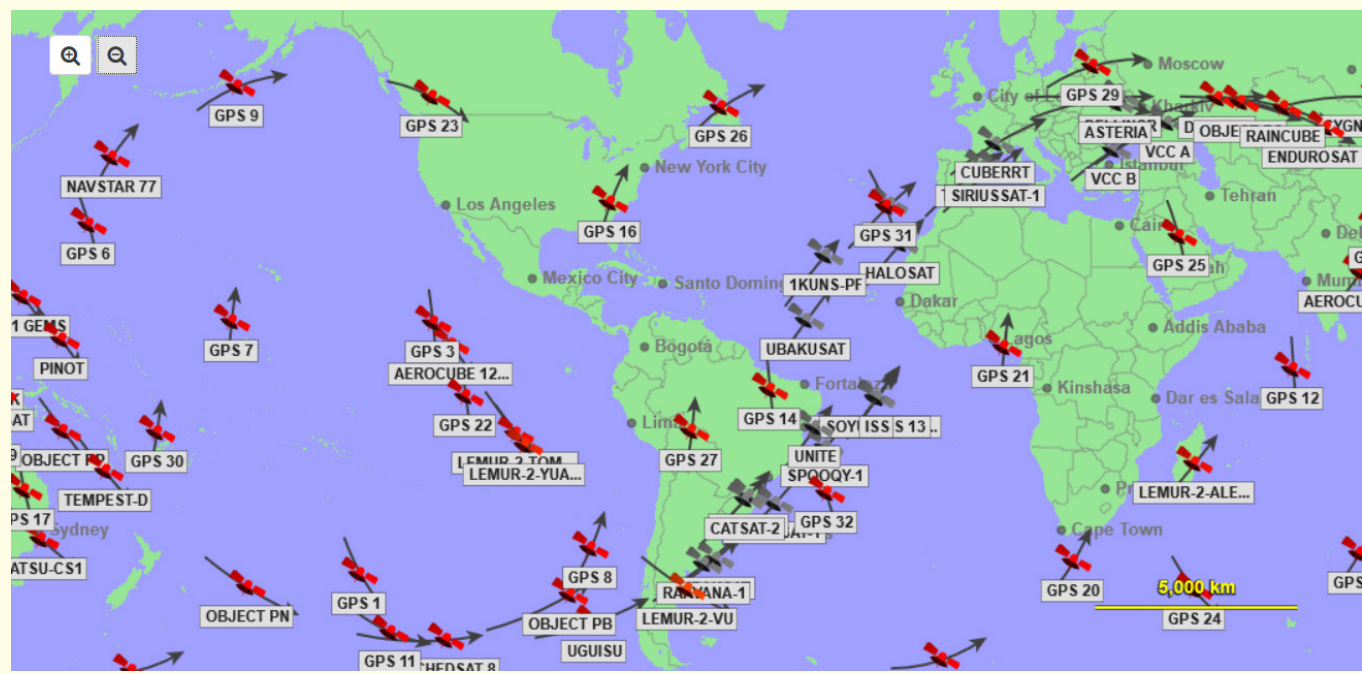

Fuente: https://in-the-sky.org/satmap_worldmap.php

En ciertos casos puede ser necesario contar con una visión más amplia de cierto fenómeno. En el mismo Atlas ya mencionado, los autores sostienen que el uso de mapas de censo de infraestructura puede ser una buena alternativa para seguir la actividad del ciberespacio en determinadas regiones. Para la cibergeografía, puntualmente, una cartografía de censo de la infraestructura de Internet en una ubicación dada, nos permite visualizar y comparar niveles de conectividad, ya sea a macro o micro escala. Si se produce un incremento en la actividad en ciertos lugares asociados a instancias de riesgo conocidas, es posible identificar nodos principales y secundarios de una red en operación que participe de la instancia de riesgo de que se trate. Dicho de otro modo, allí donde la red se encuentre más al rojo, en esos lugares es donde habrá que buscar ${ }^{20}$.

En las figuras 6 y 7 se ilustran dos reconocidos mapas de conectividad global, producidos por Larry Landweber y que evidencian la expansión de la conectividad global, entre 1991 y $1997^{21}$.

20 La anécdota del hombre que busca sus llaves bajo el cono de luz, sin saber si las perdió allí o no, viene a cuento en nuestra exposición. Las irregularidades en los patrones de uso de red, que pueden ser detectadas mediante las herramientas que nos entrega la cibergeografía, son el cono de luz bajo el cual buscaremos la red (o el conjunto de redes) maliciosas.

21 LANDWEBER, L., 1997. INTERNATIONAL CONNECTIVITY. Pages.cs.wisc.edu [online]. 1997. [Accessed 14 november 2019]. Available from: http://pages.cs.wisc.edu/ /hl/maps/ 
Figura 6

Conectividad Internacional, 1991

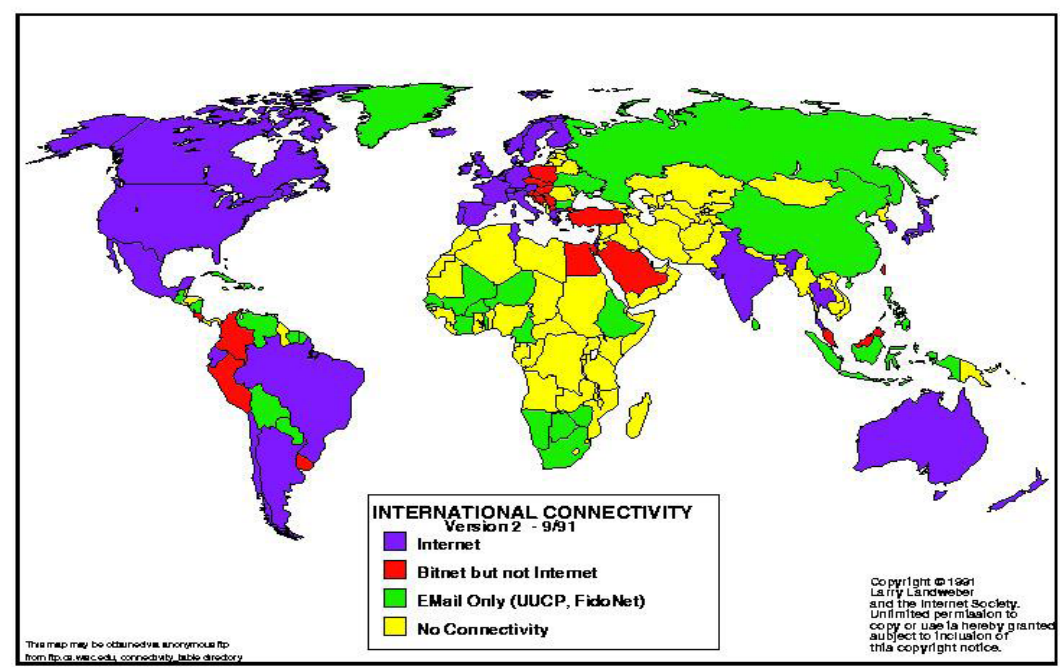

Fuente: Landweber 1997

Figura 7

Conectividad Internacional, 1997.

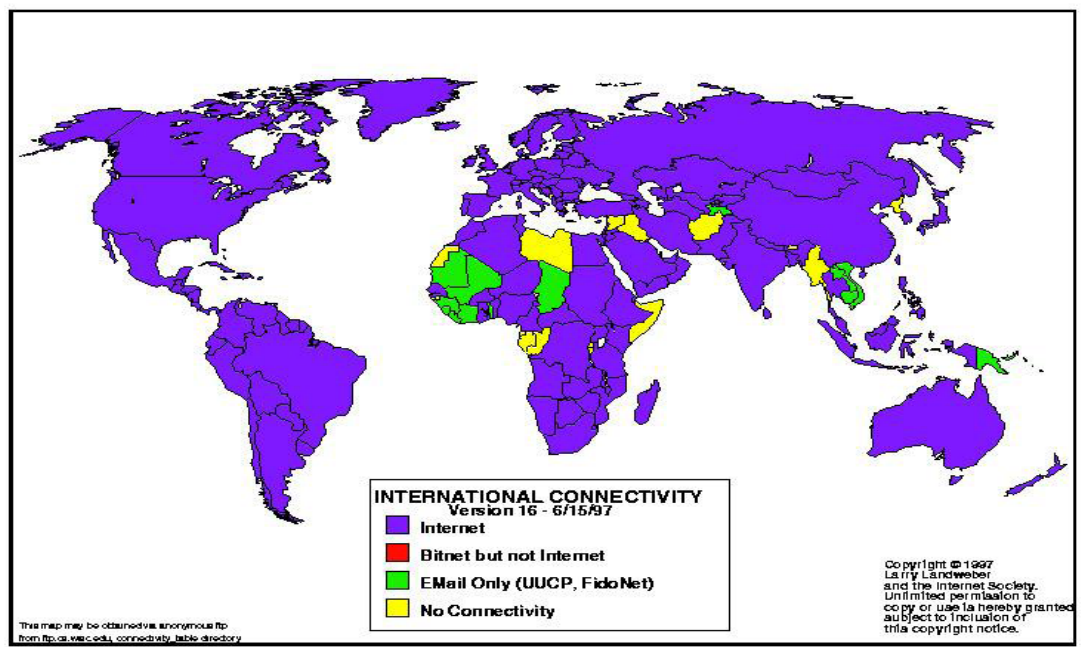

Fuente: Landweber 1997 
Este tipo de mapas nos permiten observar fácilmente cambios de regularidad a través del tiempo, lo que ciertamente apoya y sirve a la toma de decisiones. A un nivel más granular, también nos permitirían monitorear, por ejemplo, el nivel de conectividad de instalaciones dedicadas a labores de ciberdefensa en una región dada.

Profundizar en el estudio de la ubicación física de infraestructura relacionada con Internet, demanda al menos una comprensión básica de cómo un dispositivo logra alcanzar el contenido provisto por otro dispositivo en algún punto de la red. Dicho de manera resumida: un computador puede identificar a otro a través un número identificador denominado Internet protocol, más conocido como IP. Sin embargo, recordar el número IP de cada sitio web que frecuentamos, es sumamente engorroso, por lo cual se implementó el "sistema de nombres de dominio" (Domain Name System, DNS), el cual permite que podamos acceder a un sitio web a través de una frase, en lugar del número. Así, cuando usted desea acceder al portal web de la ANEPE, por ejemplo, simplemente escribe el nombre de la página, sin necesidad de conocer el número IP del computador que pone a su disposición los archivos que componen dicho portal. Para que esto sea posible, un servidor de nombres de dominio realiza la interpretación (o traduce) el nombre solicitado al número IP correspondiente.

Una explicación detallada del DNS escapa al alcance de este artículo, pero cabe acotar que el sistema consiste en una estructura jerarquizada de nodos. No obstante, saber que existen computadores que traducen frases en números, y que por lo mismo cumplen una labor tan importante para el funcionamiento de Internet como lo entendemos hoy en día, nos lleva a querer saber dónde se encuentran dichos nodos. La figura 8 ilustra la ubicación de los servidores raíz del DNS22.

Figura 8

Root Servers en el mundo

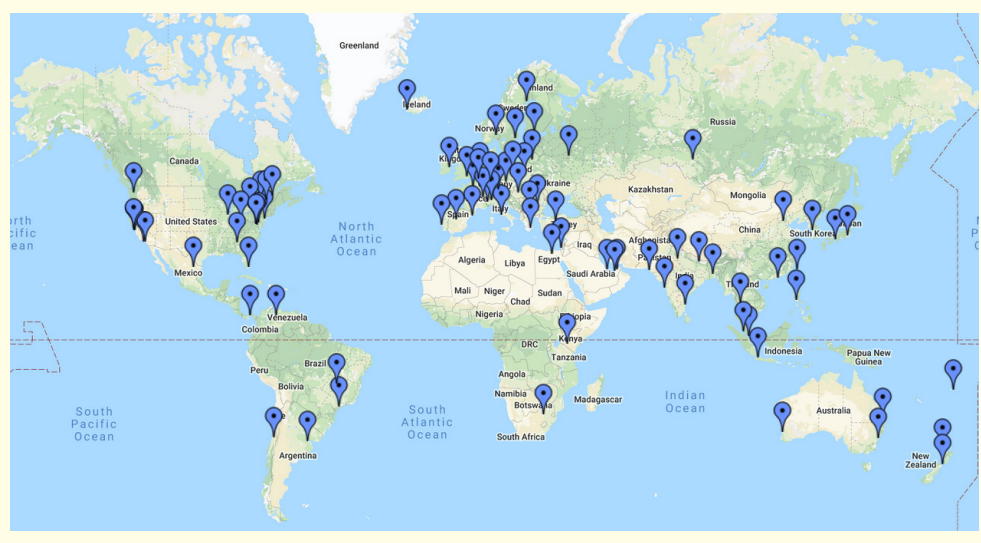

Fuente: Google Maps.

22 Root Servers in the World - Google My Maps, [n. f.]. Root Servers in the World [online], [Accessed 14 november 2019]. Available from:

https://www.google.com/maps/d/viewer?ie=UTF8\&hl=en\&msa=0\&ll=11.424428999999996\%2C26.1780 $63000000066 \&$ spn=142.883537\%2C288.632813\&z=2\&om=1\&mid=1LcHEpzl-7RzziWzDa4h3BxJcbEo 
Una inspección superficial del mapa permite identificar rápidamente la poca densidad de este tipo de servidores en América del Sur, en comparación a Europa o América del Norte.

En el segundo enfoque para la representación en la cibergeografía -el mapeo de la red-, supone un interesante desafío intelectual, considerando que convergen diversas disciplinas como la gráfica computacional, el diseño de la información, entre otras.

Uno de los primeros ejercicios mentales de este tipo, plasmados en un mapa, es el realizado por John December, quien el año 1994 publicó un mapa conceptual de los espacios de información de Internet ${ }^{23}$, el cual se expone en la figura 9.

Figura 9

Conceptual map of Internet information spaces

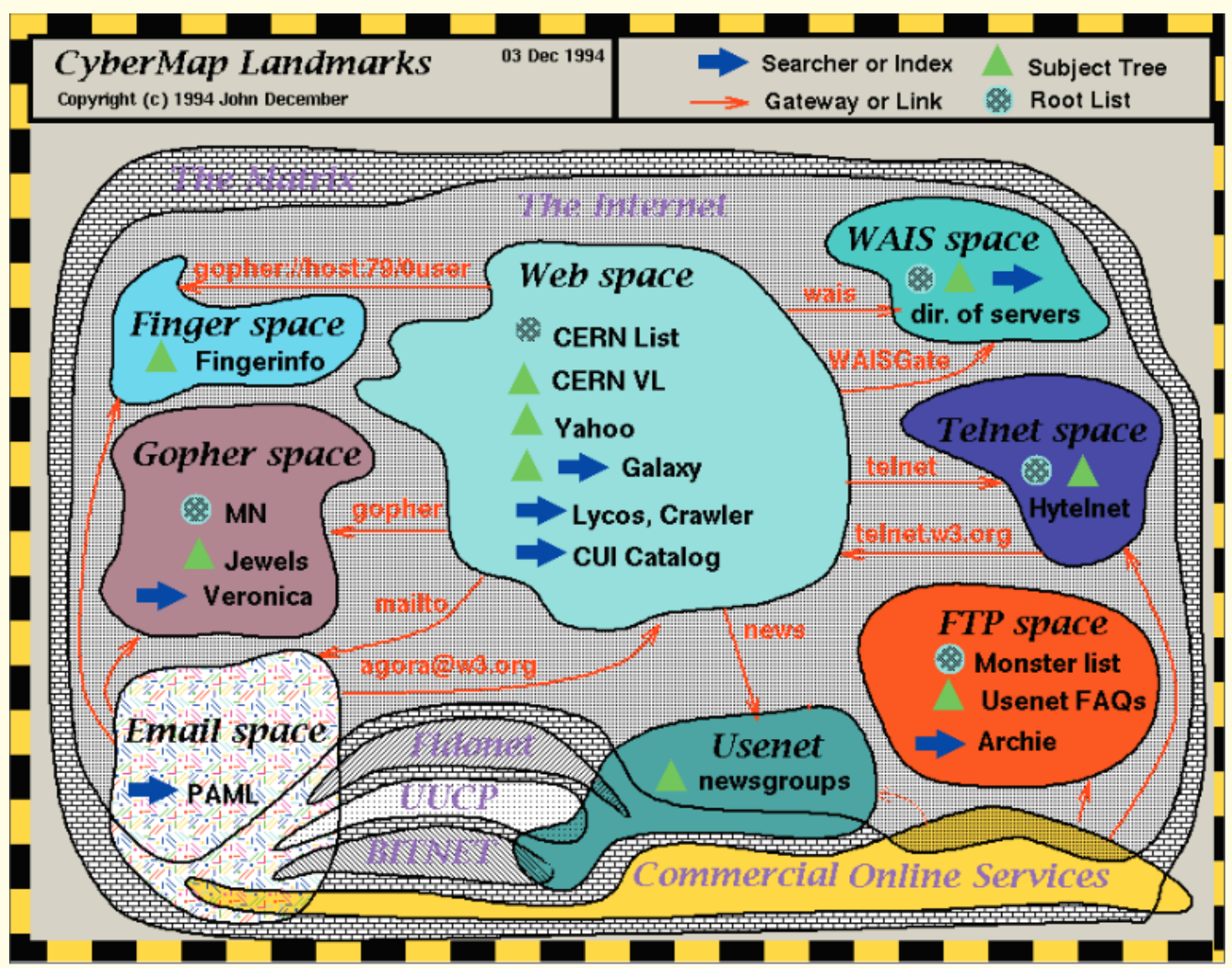

Fuente: December J., circa 1994.

Su mapa es un claro ejemplo del desafío que implica la representación cartográfica

23 DECEMBER, John. Loc. Cit. 
de fenómenos no espaciales, por cuanto la amplitud de las formas que busca representar no posee una referencia específica en el mundo físico ${ }^{24}$. Así, aunque se pueda plasmar una relación de subconjuntos entre elementos que se sabe pertenecen a alguna categoría -como, por ejemplo, el espacio compuesto por los sitios web puede considerarse parte del espacio compuesto por Internet-, el ordenamiento, magnitud y forma de los mismos quedan mucho más sujetos a la interpretación subjetiva.

En el otro extremo tenemos las representaciones de sitios web individuales. Probablemente el lector habrá notado que diversos sitios web contienen una sección denominada "mapa del sitio". Dicha sección habitualmente consta de una mera tabla de contenidos, donde se ilustran de manera plana las secciones existentes, junto con las subsecciones asociadas. Un ejemplo de esto es el mapa del sitio web del Metro de Santiago, que se muestra en la figura 10.

Figura 10

Mapa del sitio web de Metro de Santiago

\section{Mapa del Sitio}

$\begin{array}{ll}\text { Tu Viaje } & \text { Atención a Clientes } \\ \text { - Planificador } & \text { - Oficinas } \\ \text { - Conexiones } & \text { - Objetos Perdidos } \\ \text { - Plano de Red } & \text { - Tarjeta Adulto Mayor } \\ \text { - Estaciones } & \text { - Sugerencias y Reclamos } \\ \text { - Tarifas } & \text { Mreguntas Frecuentes } \\ \text { - Horarios } & \text { Metrocl } \\ \text { - Carga tu Bip! } & \text { - Mapa del Sitio } \\ \text { - Estado del Servicio } & \text { - Políticas de Privacidad } \\ \text { - Ruta Expresa } & \text { - Normas Uso y Convivencia Redes } \\ \text { Noticias } & \text { Sociales } \\ \text { - Noticias Recientes } & \text { - Trabaja con Nosotros } \\ \text { - Sala de Prensa } & \text { - Contacto al Webmaster } \\ \text { - Historias de Metro } & \\ \text { El Buen Viajero } & \text { Utilidades para funcionarios } \\ \text { - Tips de Viaje } & \text { - Acceso Preferencial } \\ \text { - Qué hacer en caso de } & \text { - Portal para Trabajadores } \\ \text { contingencia } & \text { - Verificación de Documentos } \\ \text { - Ticket de Viaje } & \end{array}$

$\begin{array}{ll}\text { Corporativo } & \text { Licitaciones } \\ \text { - Misión y Visión } & \text { - Propuestas en Curso } \\ \text { - Nuestros Valores } & \text { - Propuestas Antiguas } \\ \text { - Marco Normativo } & \text { - Registro de Empresas } \\ \text { - Objetivo o Giro de la Empresa } & \text { - Licitaciones proyecto Líneas 3 y } 6 \\ \text { - Filiales } & \text { Actividades Comerciales } \\ \text { - Estructura Organizacional } & \text { - Espacios Publicitarios } \\ \text { - Remuneraciones } & \text { - Gestión de Espacios Comerciales } \\ \text { - Información Financiera } & \text { - Permisos Comerciales } \\ \text { - Informe Financiero Anual } & \text { - Consultorías } \\ \text { - Canal de Denuncias } & \\ \text { - Reporte Operacional } & \\ \text { - Memoria } & \\ \text { - Reporte de Sostenibilidad } & \\ \text { - Historia } & \\ \text { - Mapa del Sitio } & \\ \text { - Información de Interés } & \end{array}$

Fuente: Metro de Santiago (https://www.metro.cl/corporativo/mapa-del-sitio).

El principal objetivo de estos mapas es el de proveer a los motores de búsqueda, de información concreta sobre las páginas que el sitio contiene, de modo que ellos las puedan rastrear fácilmente ${ }^{25}$. También, en el caso de los visitantes, sirven como orientadores o mapas.

24 JONGH, Charles and ORMELING, Ferjan, 2002. Mapping non-spatial phenomena. Proceedings of the Seminars on Developing the ICA-CET Internet Cartography Course, Helsinki, Finland. 2002.

25 Página principal de sitemaps.org, [no date]. Sitemaps.org [online], [Accessed 14 november 2019]. 
El último enfoque dice relación con el mapeo de las conversaciones y la comunidad. Hasta este punto los enfoques se han concentrado en los niveles físicos y lógicos del ciberespacio; sin embargo, Internet como lo conocemos hoy en día genera y facilita múltiples interacciones entre personas diariamente.

En este sentido, las redes sociales son probablemente uno de los puntos de Internet más representativos en lo que respecta a las interacciones humanas facilitadas por la red. Las figuras 11 y 12 ilustran dos ejemplos presentados por Ameel Khan en su blog personal ${ }^{26}$.

Figura 11

Red de contactos en Facebook

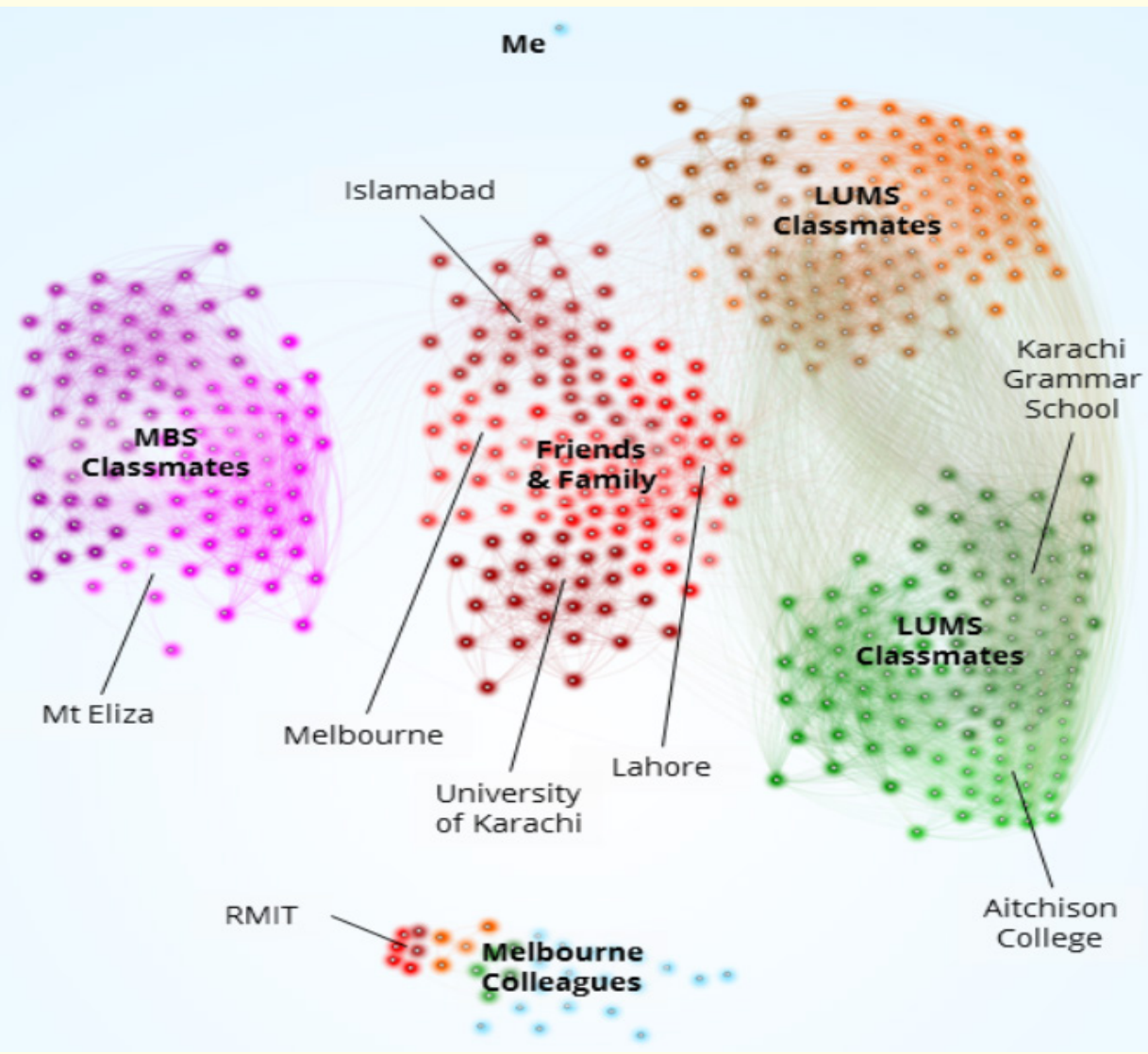

Fuente: Ameel Khan.

26 DECEMBER, John, 1994. December Communications, Inc. december.com. December.com [online]. 1994. [Accessed 14 november 2019]. Available from: https://www.december.com/ 
Figura 12

Red de contactos en LinkedIn.

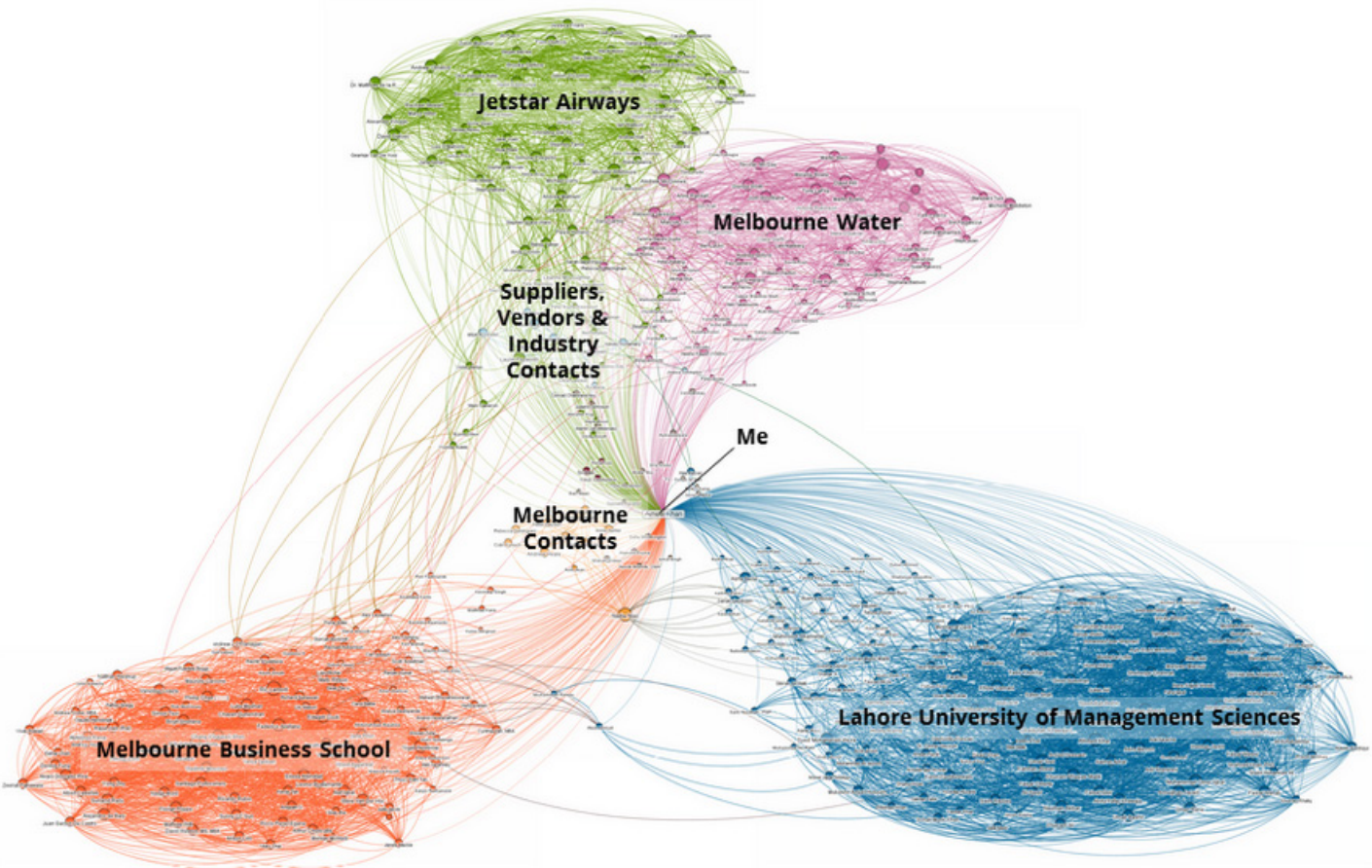

Fuente: Ameel Khan.

Este tipo de mapas permiten visualizar y explorar aspectos humanos facilitados por la tecnología, los cuales de otro modo requerirían un gran esfuerzo de abstracción.

Los enfoques que hemos visto, proveen una aproximación al heterogéneo conjunto de factores que pueden considerarse a la hora de generar mapas cibergeográficos. Los múltiples criterios posibles representan tanto problemas como oportunidades: los mapas concebidos para responder a una necesidad específica, pueden no ser apropiados para otros casos. Pero no hay que olvidar que este tipo de disyuntivas ya existen en la geografía y cartografía tradicional, con diferentes matices.

\section{RIESGOS, POSIBILIDADES Y DESAFÍOS}

Un aspecto importante para el diseño de una cibergeografía que pueda ser entendida de forma transversal por los distintos actores involucrados, es que debe existir un consenso en términos técnicos y jurídicos. Si bien hay ciertos aspectos que son universalmente entendidos, tales como los componentes físicos de una infraestructura, o los protocolos de red, entre otros, los niveles más abstractos de las definiciones se vuelven mucho más difusos; incluso poder explicar por qué los desarrollos ciberespaciales representan tecnologías significativas y transformadoras, no es tarea trivial, lo que genera 
debates académicos que pueden durar años ${ }^{27}$.

Tal disyuntiva supone riesgos tanto nacionales como internacionales, ya que del mismo modo que la persecución y el castigo de "ciberdelincuentes" requiere de un marco legal que sustente el actuar tanto de policías como de entidades judiciales, aquellos posibles conflictos y/o delitos que afecten cualquier elemento considerado dentro de la cibergeografía nacional, solo podrán ser enfrentados con el apoyo de un marco legal adecuado.

Si bien estos aspectos pueden ser relativamente simples de abordar a nivel local, ya que bastaría un consenso propio para diseñar una definición jurídica eficiente, los fenómenos e interacciones relacionadas con la red e Internet suelen involucrar a actores de diferentes países. Y aquí empiezan los problemas. La tipificación de delitos a nivel de "cibercrimen" en el país anfitrión puede ser muy diferente de aquella aplicada en el país persecutor. La falta de consenso respecto de qué constituye parte de la cibergeografía de un país (recordemos que hablamos de ciberespacio), supone un evidente riesgo de conflicto con otros países. Esto es comparable a ciertos conflictos limítrofes.

Pese a todo, esta problemática supone al mismo tiempo posibilidades interesantes para nuestro entendimiento del mundo interconectado de hoy. La cibergeografía en cierta medida apunta a un orden en los límites del ciberespacio, lo cual puede favorecer o al menos facilitar el trabajo colaborativo entre países en lo que respecta a la infraestructura física y virtual que sostienen las interacciones digitales de hoy. Ya se trate de la identificación predictiva de espacios que requieren mejoras en su infraestructura, ya de la protección del ciberespacio relativo a un sector cibergeográfico específico, la dimensión espacial puede aportar tanto en la búsqueda de acuerdos como en la prevención de delitos o ilícitos basados en Internet.

El desafío ante las tendencias disruptivas y las tecnologías de la información, para el desarrollo de las operaciones militares, es sin duda la dimensión del ciberespacio que más escenarios abarca ${ }^{28}$, tal como se plantea en la investigación "La Dimensión del Ciberespacio: una propuesta de ciberseguridad". Sin locación física específica, la idea del agresor o del enemigo, obliga a replantearse la categorías tradicionales de la "guerra real" y exige, por la dinámica propia de la innovación tecnológica, una rápida adaptación para los Sistemas de Defensa respecto de sus componentes. En las últimas décadas, muchos países vienen reorientando esfuerzos y recursos para resguardar no solo los espacios tradicionales (terrestre, marítimo y aeroespacial), sino también el ciberespacio. Es aquí, precisamente, donde la cibergeografía, de momento, al menos, es la única posibilidad real.

27 KITCHIN, Robert M. Loc. Cit.

28 BARRÍA, Cristian, 2019. La Dimensión del Ciberespacio: una propuesta de ciberseguridad. Cuadernos de Trabajo, Centro de Investigaciones y Estudios Estratégicos (CIEE) [online]. 2019. No. 1. [Accessed 14 november 2019]. Available from: https://www.anepe.cl/centro-de-estudios-estrategicos-2/cuadernos-detrabajo/ 


\section{CIBERGEOGRAFÍA Y LA SEGURIDAD NACIONAL}

La geolocalización dentro de la cibergeografía reviste una gran importancia, por su impacto en el espacio físico, ya que es allí donde se manifiesta la vida social de los hombres. Tal como se ha señalado, este nuevo ámbito de circulación de la información no constituye un espacio en sí mismo, un espacio a la manera tradicional, sino más bien una dimensión superpuesta que traspasa los espacios físicos tradicionales, y que resulta de vital importancia para comprender las implicancias del mismo en el ámbito de la Defensa, ya que las operaciones virtuales -entendidas como operaciones de información- resultan de interés para los Estados por su capacidad de producir alteraciones y/o modificaciones en el mundo físico ${ }^{29}$.

En su libro "Reflexiones sobre la Geografía Estratégica Militar 2.0", Jane Holloway expone la relevancia que ha alcanzado la geografía en los asuntos militares modernos, destacando la cibergeografía como una de las dimensiones clave en una fuerza de Defensa que busque estar a la par con los nuevos desafíos que impone el complejo mundo interconectado de hoy ${ }^{30}$.

Dentro de su propuesta marco de trabajo para la modernización de la geografía militar (SMG 2.0), Holloway plantea la necesidad de reconocer esta asignatura como una nueva subdisciplina y como un ámbito geográfico propio, el cual aporte distintos aspectos técnicos y tecnológicos que permitan y expandan nuestro conocimiento espacial, temporal y ambiental, elementos fundamentales para generar una capacidad de respuesta estratégica y operativa, oportuna y eficiente.

Toda esta "ciber esfera", sostiene Holloway, ha despertado un explosivo interés en las organizaciones de Defensa, en las cuales se puede apreciar un aumento en las investigaciones sobre tópicos como la ciberseguridad y ciberguerra ${ }^{31}$. $Y$ no es algo que debiera sorprendernos, considerando los diversos incidentes de ciberseguridad que afectan a todo el mundo, tanto a la comunidad civil como a la militar. Y Chile no es la excepción.

\section{DISYUNTIVAS RELACIONADAS CON LA CIBERGEOGRAFÍA}

Algunos de los principales cuestionamientos a esta dimensión son de tipo valórico. La geografía, y la cibergeografía (o sea, la geografía del ciberespacio), es acerca de datos. Los datos revelan cosas que relaciona a personas. $Y$ las partes que revelan no son pocas o triviales. Al conocer aspectos como conectividad, intensidad de tráfico, orígenes y destinos de los mensajes, calidad y confiabilidad de los mismos, etc., los encargados de realizar estos trabajos (aun cuando se trate de algoritmos) deben inmiscuirse en las vidas de las personas. Este escenario es y representará un problema.

29 DE VERGARA, Evergisto, TRAMA, Gustavo Adolfo, URIONA, Marcelo Noel, ORTIZ, Javier Ulises and DESTRO, Lucía Alejandra, 2018. Operaciones militares cibernéticas: Planeamiento y Ejecución en el Nivel Operacional. Editorial Visión Conjunta.

30 HOLLOWAY, Jane L., 2018. Reflections on Strategic Military Geography 2.0. Advances in Military Geosciences. 2018. pp. 49-67. DOI 10.1007/978-3-319-73408-8_4. Springer International Publishing

31 SINGER, P. W and FRIEDMAN, Allan, 2014. Cybersecurity and cyberwar. New York: Oxford University Press. 
Las amenazas del ciberespacio no son ajenas a institución alguna, sin importar si estas son públicas o privadas, civiles o militares. Desde un usuario común hasta un gobierno completo, encontrarán en el ciberespacio toda clase de posibles atacantes que buscarán atentar contra sus intereses. Esto naturalmente motiva la discusión e implementación de medidas preventivas y/o de respuesta ante estas amenazas, con el fin de resguardar aquellos datos y activos de interés. Pero en el legítimo afán de proteger surgen ciertas necesidades controvertidas, como el poder identificar a quien está del otro lado de la pantalla.

Si bien en un escenario en el que del "otro lado" hay efectivamente un ciberdelincuente, para poder determinar que lo sea hace falta un amplio trabajo de investigación y, en ocasiones, de monitoreo. En este trabajo se busca, de una u otra forma, individualizar a ese atacante que hasta entonces, es una entidad anónima. Durante esa individualización la geolocalización sin duda provee de una poderosa herramienta para que las entidades respectivas puedan dar con el paradero del delincuente.

Sin embargo, supone un escenario ideal en el que el sospechoso es realmente el atacante. Si bien los conceptos técnicos y jurídicos de estas situaciones escapan al alcance de este trabajo, es importante acotar que un ecosistema de geolocalización potenciada y mejorada por trabajos tecnológicos de cibergeografía, podría cruzar la línea que separe lo público de lo privado.

Si se llega a implementar una infraestructura perfectamente (ciber) cartografiable, con datos en tiempo real, que alimenten sistemas de monitoreo y/o de seguimiento, ¿es aceptable que aquellas personas que cumplen con la ley deban ser monitoreadas? Otros cuestionamientos surgen con facilidad: ¿qué ocurrirá en los casos en los que una investigación digital, decante en el enfrentamiento físico de personas, producto de una sospecha equivocada? ¿Quién asumirá la responsabilidad, en caso de una filtración de datos de geolocalización de personas inocentes?

Es evidente que la seguridad nacional requiere realizar ciertas tareas de inteligencia que buscan proteger al país, pero, así como ha ocurrido con otras tecnologías, un desacierto o un descuido podría ocasionar un amplio rechazo en las personas frustrando los intereses legítimos de la seguridad nacional. Es, entonces, deber de las organizaciones respectivas velar por un cuidadoso estudio e implementación de cualquier tecnología de este tipo, para no transgredir la privacidad de las personas.

Si bien la cibergeografía por sí misma no es causante ni responsable de este tipo de problemas, su interacción con otras tecnologías y/o disciplinas tienen importantes riesgos asociados, riesgos que pueden abordarse con antelación de modo de evitar errores que frustren futuras iniciativas.

\section{CONCLUSIONES}

En las páginas precedentes hemos presentado un resumen bastante acotado de esta incipiente disciplina llamada cibergeografía. Hemos abordado distintos casos históricos que abren las puertas para tratar algunos temas relacionados, tales como la seguridad 
nacional y la geolocalización.

Esta disciplina tiene un largo camino por recorrer, un trayecto que, bien llevado, contribuirá a nuestro entendimiento del ciberespacio, así como a una mejora importante en las representaciones que realizamos de aspectos como infraestructura y comunicaciones, entre otros.

En un mundo ya altamente interconectado, en donde Internet toma un rol protagónico, poder levantar representaciones cartográficas de los elementos que lo conforman y que son generados por él, será una necesidad insoslayable. Trazar esta cartografía no será una opción, sino una demanda que con el tiempo irá incrementando. Asimismo, todo progreso técnico trae aparejados conflictos que antes no existían.

La valoración que hagamos de los mismos importará conocer sus perímetros y sus alcances éticos. No será tarea sencilla, pero tampoco será posible soslayarla. Las prestaciones de Internet son parte de nuestra cultura; esto es, dependemos de ellas para vivir, por lo tanto el desafío, como decimos, es insoslayable.

\section{REFERENCIAS BIBLIOGRÁFICAS}

BARR, Chas. B., 1853. Telegraph stations in the United States, the Canadas \& Nova Scotia. [S.I] [Map] [online]. [image]. 1853. [Accessed 14 november 2019]. Available from: https://www.loc.gov/item/97683602/

BARRÍA, Cristian, 2019. La Dimensión del Ciberespacio: una propuesta de ciberseguridad. Cuadernos de Trabajo, Centro de Investigaciones y Estudios Estratégicos (CIEE) [online]. 2019. No. 1. [Accessed 14 november 2019]. Available from: https://www. anepe.cl/centro-de-estudios-estrategicos-2/cuadernos-de-trabajo/

BUZAI, Gustavo D., 2001. Paradigma Geotecnológico, Geografía Global y CiberGeografía, la gran explosión de un universo digital en expansión. GeoFocus. Revista Internacional de Ciencia y Tecnología de la Información Geográfica. 2001. Vol. 1, pp. 24-48.

BUZAI, Gustavo, 2001. Cibergeografía y la destrucción del "mito" de la red mundial sin centro. Estudios socioterritoriales. 2001. Vol. 2, no. 2, pp. 9-24.

CERF, V and KAHN, B, 1990. Selected ARPANET maps. Computer Communications Review (CCR). 1990. No. 20, pp. 81-110.

DECEMBER, John, 1994. December Communications, Inc. december.com. December. com [online]. 1994. [Accessed 14 november 2019]. Available from: https://www. december.com/

DODGE, Martin and KITCHIN, Rob, 2001. Atlas of cyberspace. Harlow: Pearson Education.

FELIÚ Ortega, Luis, 2012. La confusa terminología de la seguridad y la defensa. IEEE, Documento de opinión. 2012. p. 6. 
FIDLER, Bradley and CURRIE, Morgan, 2015. The Production and Interpretation of ARPANET Maps. IEEE Annals of the History of Computing. 2015. Vol. 37, no. 1, pp. 44-55. DOI 10.1109/mahc.2015.16. Institute of Electrical and Electronics Engineers (IEEE)

FORD, Dominic, 2019. Live World Map of Satellite Positions - In-The-Sky.org. In-the-sky.org [online]. 2019. [Accessed 14 november 2019]. Available from: https://in-the-sky. org/satmap_worldmap.php

HOLLOWAY, Jane L., 2018. Reflections on Strategic Military Geography 2.0. Advances in Military Geosciences. 2018. pp. 49-67. DOI 10.1007/978-3-319-73408-8_4. Springer International Publishing

JONGH, Charles and ORMELING, Ferjan, 2002. Mapping non-spatial phenomena. Proceedings of the Seminars on Developing the ICA-CET Internet Cartography Course, Helsinki, Finland. 2002.

KHAN, Ameel, 2013. Mapping My Social Networks: Facebook, LinkedIn. Random Tangent [online]. 2013. [Accessed 14 november 2019]. Available from: https://www. insanityworks.org/randomtangent/2013/7/18/mapping-my-social-networksfacebook-linkedin

KITCHIN, Robert M., 1998. Towards geographies of cyberspace. Progress in Human Geography. 1998. Vol. 22, no. 3, pp. 385-406. DOI 10.1191/030913298668331585. SAGE Publications

LANDWEBER, L., 1997. INTERNATIONAL CONNECTIVITY. Pages.cs.wisc.edu [online]. 1997. [Accessed 14 november 2019]. Available from: http://pages.cs.wisc.edu/ /hl/maps/

Página principal de sitemaps.org, [no date]. Sitemaps.org [online],

RAMÍREZ Velázquez, Blanca Rebeca and LÓPEZ Levi, Liliana, 2015. Espacio, paisaje, región, territorio y lugar. 1. Geografía para el siglo XXI: Serie Textos universitarios.

Root Servers in the World - Google My Maps, [no date]. Root Servers in the World [online],

SAGAN, Carl, 2000. Carl Sagan's cosmic connection. Cambridge: Cambridge University Press.

SIERRA, Daniel, 2015. Las dos caras de la tecnología. CIBER Elcano [online]. 2015. No. 2, p. 15. [Accessed 14 november 2019]. Available from: http://www.realinstitutoelcano. org/wps/wcm/connect/6df2110047ec9efab8bbbe8dbc02bac7/Ciber_Elcano_ Num2.pdf?MOD=AJPERES\&CACHEID=1428393273980

SINGER, P. W and FRIEDMAN, Allan, 2014. Cybersecurity and cyberwar. New York: Oxford University Press.

UMPLEBY, Stuart A. y DENT, Eric B., 1999. THE ORIGINS AND PURPOSES OF SEVERAL 
TRADITIONS IN SYSTEMS THEORY AND CYBERNETICS. Cybernetics and Systems. 1999. Vol. 30, no. 2, pp. 79-103. DOI 10.1080/019697299125299. Informa UK Limited

Submarine Cable Map, [no date]. https://www.submarinecablemap.com/ [online],

VAN CREVELD, MARTIN, 2010. Technology and war: From 2000 BC to the present. Simon and Schuster.

DE VERGARA, Evergisto, TRAMA, Gustavo Adolfo, URIONA, Marcelo Noel, ORTIZ, Javier Ulises and DESTRO, Lucía Alejandra, 2018. Operaciones militares cibernéticas: Planeamiento y Ejecución en el Nivel Operacional. Editorial Visión Conjunta.

WILLIAMS, BRETT, 2019.. Cyberspace: What is it, where is it and who cares? Armed Forces Journal [online]. 2019. [Accessed 14 november 2019]. Available from: http:// armedforcesjournal.com/cyberspace-what-is-it-where-is-it-and-who-cares/ 